\title{
ON FREE PRODUCTS ${ }^{1}$
}

\section{A. KARRASS AND D. SOLITAR}

We prove several theorems for free products most of which are generalizations of results known for free groups.

Let $G$ be a nontrivial free product of finitely many groups $A_{i}$ each of which is a finite extension of a free group $F_{i}$ of finite rank $\geqq 0$. Then we call $G$ a free product of finite type. In particular a free product of finitely many finite groups is of finite type. In what follows "f.i." denotes "finite index" and "f.g." denotes "finitely generated."

Theorem 1. Let $G$ be a free product of finite type and $H \neq 1, a f . g$. subgroup of $G$. If $H$ is normal (or more generally if $H$ contains a nontrivial normal subgroup of $G$ ) then $H$ is of f.i. in $G$.

Proof. Let $F$ be the normal subgroup of $G$ generated by the $F_{i}$ and the commutators $\left[A_{i}, A_{j}\right](i \neq j)$. We show $F$ is a free group. For, by the Kurosh Subgroup Theorem (see [6]), $F$ is the free product of a free group and the intersections of $F$ with various conjugates of the $A_{i}$. The intersection of $F$ with a conjugate of $A_{i}$ is a conjugate of $F \cap A_{i}$. It therefore suffices to show $F \cap A_{i}$ is free. Consider the natural homomorphism $\nu$ of $G$ onto $G / F$. Now $G / F$ is isomorphic to the direct product of the groups $A_{i} / F_{i}$. Moreover, under $\nu, A_{i}$ is mapped canonically onto $A_{i} / F_{i}$. Hence $F \cap A_{i}$, which is the kernel of $\nu$ restricted to $A_{i}$, must be $F_{i}$. Clearly then $F$ is a free group of f.i. in $G$.

Let $N \neq 1$ be a normal subgroup of $G$ contained in $H$. Then $N$ must be infinite. For otherwise, applying the Kurosh Subgroup Theorem, we would have $N$ contained in a conjugate of some $A_{i}$. But then the normalizer of $N$ would also be in this conjugate (see proof of Theorem 5), contrary to $N$ being normal in $G$. By the isomorphism theorem $N / N \cap F \simeq N F / F$ and $H / H \cap F \simeq H F / F$. Hence $N \cap F$ is of f.i. in $N$ so that $N \cap F \neq 1$. Also $H \cap F$ is of f.i. in the f.g. group $H$, and so $H \cap F$ is f.g. (see Schreier [9]). Thus we have $H \cap F$ is a f.g. subgroup of a free group $G$ containing $N \cap F$, a nontrivial normal subgroup of $F$. Consequently (see [5]), $H \cap F$ is of f.i. in $F$ and so $H$ is of f.i. in $G$.

Corollary 1. Let $G$ be a free product of finite type, and $H$ a f.g.

Presented to the society, April 5, 1957 ; received by the editors October 9, 1957.

1 Work on this paper was supported by a grant from the National Science Foundation (G-2796). 
subgroup. If $H$ is of infinite index then the intersection of the conjugates of $H$ must be 1 .

Corollary 2. If $G$ is a free product of finite type, and $H_{1}, H_{2}$ are f.g. subgroups, then $\mathrm{H}_{1} \cap H_{2}$ is f.g.

Proof. First observe that if $G$ is any group, $K$ a subgroup of $f . i$, II a f.g. subgroup, then $H \cap K$ is f.g., since $H \cap K$ is of f.i. in $H$.

Now let $F$ be as in the proof of Theorem 1. Then $H_{1} \cap F, H_{2} \cap F$ are f.g. subgroups of the free group $F$ and therefore $H_{1} \cap H_{2} \cap F$ is f.g. (see Howson [4]), and is of f.i. in $H_{1} \cap H_{2}$. Hence a set of generators for $H_{1} \cap H_{2} \cap F$ together with coset representatives for $H_{1} \cap H_{2}$ $\bmod H_{1} \cap H_{2} \cap F$ constitutes a finite set of generators for $H_{1} \cap H_{2}$.

In particular, taking $G$ to be the modular group, we see that the intersection of two f.g. groups of unimodular $2 \times 2$ integral matrices is $\mathrm{f} . \mathrm{g}$.

THEOREM 2. If $G$ is a free product of finite type, then $G$ is hopfian, i.e. $G \simeq G / N$ implies $N=1$.

Proof. Let $G_{k}$ denote the intersection of all subgroups of $G$ of in$\operatorname{dex} \leqq k$. Then $\bigcap_{1}^{\infty} G_{k}=1$. For if $F$ is as above, then the intersection of the subgroups of f.i. in $F$ (and therefore in $G$ ) is 1 (see e.g. Kurosh [6]). Let $G \simeq G / N$ and let $\nu$ be the natural homomorphism of $G$ onto $G / N$. Since $G$ is f.g. it has only finitely many subgroups of f.i. $\leqq k$ (see Hall [2]) and the number of these is the same as the number of subgroups of f.i. $\leqq k$ in $G / N$. But the pre-image under $\nu$ of such a subgroup of $G / N$ is a subgroup of $G$, of f.i. $\leqq k$, and containing $N$. Hence $G_{k} \supset N$ for each $k$, and so $N=1$.

COROLlary 3. If $G$ is any f.g. group such that the intersection of its subgroups of $f . i$. is 1 , then $G$ is hopfian.

Corollary 4. Let $G$ be any f.g. group and let $N$ be the intersection of its subgroups of $f . i$. Then $G / N$ is hopfian, and moreover $G / N \simeq G / K$ implies $N=K$.

Proof. Clearly the intersection of the subgroups of f.i. in $G / N$ is 1 , and so $G / N$ is hopfian. Moreover, $G / N$ and therefore $G / K$, have as many subgroups of f.i. $m$ as $G$ has. Hence, as in the proof above, each subgroup of f.i. in $G$ contains $K$. Therefore $N \supset K$ so that $G / K \simeq G / N \simeq(G / K) /(N / K)$. But since $G / N$ is hopfian, $G / K$ is hopfian and so $N=K$.

That Theorem 2 does not hold for any f.g. nontrivial free product follows immediately from the existence of a f.g. non-hopfian group $H$ (see Neumann [8] or Higman [3]). For, let $H \simeq H / K, K \neq 1$. If 
$G=H * L, L$ any f.g. group and $N$ the normal subgroup of $G$ generated by $K$, then $G / N \simeq(H / K) * L \simeq H * L=G$.

A group $G$ is $\omega$-nilpotent ( $\omega$-solvable) if the intersection of the groups of the lower central series (derived series) is the identity. Next a generalization of a theorem of W. Magnus.

Theorem 3. If $G$ is a free product of finite type and each $A_{i}$ is a prime power extension of $F_{i}$ corresponding to the same prime, then $G$ is $\omega$-nilpotent. Moreover, if the $A_{i}$ are finite, then $G$ is $\omega$-nilpotent if and only if the $A_{i}$ are prime power groups corresponding to the same prime.

Proof. Let $F$ be as above. If the $A_{i} / F_{i}$ are $p$-groups, then $G / F$ is a $p$-group. Form the groups $Q_{0}=F, Q_{j+1}=$ the intersection of all normal subgroups of index $p$ in $Q_{j}$. We may now proceed as for free groups (see Hall [2]) to obtain $\cap Q_{j}=1$ and that the intersection of the groups of the lower central series of $G$ is 1 .

Now, if some $A_{i}$ has an element of order $p$, and some $A_{j}(j \neq i)$ has an element of order $q, p, q$ distinct primes, then $G$ has a subgroup which is the free product of a cyclic group of order $p$ by a cyclic group of order $q$. This group is not $\omega$-nilpotent (see Takahasi [10]). Hence $G$ cannot be $\omega$-nilpotent. In particular if the $A_{i}$ are finite, they must all be $p$-groups.

Theorem 4. If $G$ is a free product of finite type and each $A_{i} / F_{i}$ is solvable, then $G$ is $\omega$-solvable. Moreover, if the $A_{i}$ are finite, then $G$ is $\omega$-solvable if and only if the $A_{i}$ are solvable.

Proof. Let $F$ be as above. Then $G / F$ is solvable and $F$ is $\omega$ solvable (since it is free). Hence the $k$ th group $G^{(k)}$ of the derived series of $G$ is in $F$ so that $G^{(k+n)} \subset F^{(n)}$. Thus $G$ is $\omega$-solvable. Moreover, if $G$ is $\omega$-solvable, each subgroup is $\omega$-solvable, and therefore $A_{i}$ finite implies $A_{i}$ solvable.

TheoRem 5. Let $G$ be an arbitrary nontrivial free product and $H^{(\neq 1)}$ a subgroup having finitely many conjugates $H_{i}$. Then $\cap H_{i} \neq 1$.

Proof. Let $A$ be one of the factors of $G$, and let $a \in A, a \neq 1, v \in G$. Then $v^{-1} a v \in A$ implies $v \in A$. For, let $v=b_{1} b_{2} \cdots b_{r}$ be the reduced form of $v$ in the free product $G$. Then $v^{-1} a v$ will have syllable length $>1$ unless $b_{1} \in A$. In that case $b_{1}^{-1} a b_{1} \in A$, and its conjugate by $b_{2} \cdots b_{r}$ is in $A$. Therefore, by induction, $b_{2} \cdots b_{r}$ and so $v$ must be in $A$. Consequently, the normalizer of a subset of a conjugate of $A$ must be in that conjugate of $A$.

Moreover, if $h$ is not in a conjugate $C_{j}$ of a factor of $G$, then the normalizer $N_{h}$ of $h$ is infinite cyclic. For, the preceding remark implies 
that $N_{h}$ is disjoint from the $C_{j}$, which in turn implies (by the Kurosh Subgroup Theorem) that $N_{h}$ is a free group. Since every element of $N_{h}$ commutes with $h, h$ must be in each cyclic free factor of $N_{l}$. Therefore, $N_{h}$ is infinite cyclic.

Next, suppose $H, K(\neq 1)$ are normal subgroups of $S$, a subgroup of $G$ not contained in any $C_{j}$. Then $I \cap K \neq 1$. For otherwise, each element of $I I$ commutes with each element of $K$. If $H$ intersects some $\mathrm{C}_{j}$ in more than the identity, then $K \subset C_{j}$ and therefore $S \subset C_{j}$. Hence $\exists h \in H$, with $h$ in no $C_{j}$, and $K \subset N_{h}$. Then $K$ and the cyclic group generated by $h$ intersect nontrivially, since $N_{h}$ is infinite cyclic. Thus $H \cap K \neq 1$.

Finally, let $H_{1}, \cdots, H_{n}$ be the conjugates of $H$. Now $N_{H_{i}}$ (the normalizer of $H_{i}$ ) is of f.i. in $G$ and so $N=\bigcap_{1}^{n} N_{H_{i}}$ is of f.i. in $G$. Since $H_{i}$ is infinite, $N \cap H_{i} \neq 1$. But since $N$ is in no $C_{j}\left(C_{j}\right.$ cannot be of f.i. in $G$ ) we can apply the remark of the preceding paragraph to obtain $\bigcap_{1}^{n} H_{i}=\bigcap^{n}\left(N \cap H_{i}\right) \neq 1$.

Corollary 5. Let $G$ be a free product of finite type and let $H$ be a f.g. subgroup. Then the following three conditions are equivalent:

(a) $H$ is of $f . i$,

(b) $H$ has finitely many conjugates, and

(c) $H$ contains the dth powers of all elements of $G$ for some $d>0$.

Proof. That (a) and (b) are equivalent follows from Theorems 1 and 5. For the equivalence of (a) and (c) see [5].

CoRollary 6. Let $G$ be a free product of cyclic groups. If two elements of $G$ commute then they must be powers of the same element.

In particular, taking $G$ to be the modular group, it follows that if two unimodular $2 \times 2$ integral matrices commute then they must be powers of the same unimodular matrix. This result was first proved by K. Goldberg [1] and later generalized by O. Taussky and J. Todd [11] to matrices over a complex quadratic field. The methods used in both papers are matrix methods.

That a non-normal subgroup $H$ of a free product can be of infinite index and yet have only finitely many conjugates can be seen as follows: Let $F$ be the free group on $a, b$, and let $K$ be the subgroup of index 2 in $F$ consisting of all words of even length. It can be shown that $K$ is freely generated by $a^{2}, a b, b a$. Let $H$ be the normal subgroup of $K$ generated by $a b$. Clearly $K: H$, and hence $F: H$ is infinite. Now $a^{-1}(a b) a=b a$ is not in $H$. Therefore the normalizer of $H$ in $F$ is just $K$. Thus $H$ is of infinite index in $F$ and yet has only two conjugates. 
Let $G$ be an extension of a f.g. free group $F$ by a finite group. Using the same methods we could conclude: $G$ is hopfian; $G / F$ is a $p$-group implies $G$ is $\omega$-nilpotent; $G / F$ is solvable implies $G$ is $\omega$-solvable. Theorems 1 and 5 hold for $G$ if we assume the subgroup $H$ is infinite. This last remark implies that the Braid Group can not be a finite extension of a free group, since it contains a f.g. infinite normal subgroup of infinite index, namely the normal divisor $N$, the quotient group of which is the mapping-class group of a sphere with $n$ holes, $n$ being the degree of the Braid Group. (See Magnus [7].)

\section{REFERENCES}

1. K. Goldberg, Unimodular matrices of order 2 that commute, Journal of the Washington Academy of Sciences vol. 46 (1956) pp. 337-338.

2. M. Hall, Jr., A topology for free groups and related groups, Ann. of Math. vol. 52 (1950) pp. 127-139.

3. G. Higman, $A$ finitely related group with an isomorphic proper factor group, J. London Math. Soc. vol. 26 (1951) pp. 59-61.

4. A. G. Howson, On the intersection of finitely generated free groups, J. London Math. Soc. vol. 29 (1954) pp. 428-434.

5. A. Karrass and D. Solitar, Note on a theorem of Schreier, Proc. Amer. Math. Soc. vol. 8 (1957) pp. 696-697.

6. A. G. Kurosh, The theory of groups (2), New York, Chelsea, 1955, pp. 17-26, pp. $42-43$.

7. W. Magnus, Veber Automorphismen von Fundamentalgruppen berandeter Flaechen, Math. Ann. vol. 109 (1934) pp. 617-646.

8. B. H. Neumann, $A$ two-generator group isomorphic to a proper factor group, J. London Math. Soc. vol. 25 (1950) pp. 247-248.

9. O. Schreier, Die Untergruppen der freien gruppen, Hamberg Abh. vol. 5 (1927) pp. 161-183.

10. M. Takahasi, Note on word subgroups in free products of groups, Journal of the Institute of Polytechnics Osaka City University Series A. vol. 2 (1951) pp. 13-18.

11. O. Taussky and J. Todd, Commuting bilinear transformations and matrices, Journal of the Washington Academy of Sciences vol. 46 (1956) pp. 373-375.

Brooklyn College 